\title{
Open sublocales of localic completions
}

\author{
ERIK PALMGREN ${ }^{1}$
}

\begin{abstract}
We give a constructive characterization of morphisms between open sublocales of localic completions of locally compact metric (LCM) spaces, in terms of continuous functions. The category of open subspaces of LCM spaces is thereby shown to embed fully faithfully into the category of locales (or formal topologies).
\end{abstract}

2000 Mathematics Subject Classification 03F60, 18B30, 54E99 (primary)

Keywords: Locales, formal topologies, locally compact metric spaces

\section{Introduction}

This paper is part of the general programme to relate constructive analysis and topology in the sense of Bishop [1] to developments based on point-free topology [2, 6].

As is well-known the standard adjunction between locales and topological spaces [2] gives a fully faithful embedding of the category of sober spaces (which includes Hausdorff spaces) into locales

$$
\Omega: \text { Sob } \longrightarrow \text { Locales. }
$$

From a strict constructive point of view this embedding of categories is of little use since it cannot be proved, without employing axioms such as the Fan Theorem, that $\Omega(\mathbb{R})$ is isomorphic to the localic reals $\mathcal{R}$. For this reason one considers a different and more restricted embedding which sends $\mathbb{R}$ to a locale isomorphic to $\mathcal{R}$. In two previous papers $[4,5]$ we studied the embedding of locally compact metric spaces (in the sense of [1]) into locales or formal topologies. Vickers' construction of the localic completion of a metric space $[8,9]$ gives rise to a full and faithful functor $M:$ LCM $\longrightarrow$ FTop from the category of locally compact metric (LCM) spaces in to the category of (inductively generated) formal topologies; see [4]. This means, in

\footnotetext{
${ }^{1}$ The author was supported by research grants from the Swedish Research Council (VR) and the Swedish Collegium for Advanced Study (SCAS), and a travel grant from Japan Advanced Institute of Science and Technology (JAIST).
} 
particular, that there is a bijection between the continuous maps $X \longrightarrow Y$ (i.e. locally uniformly continuous functions) and the continuous morphisms of formal topologies $M(X) \longrightarrow M(Y)$ (approximable mappings).

To study point-free versions of topological manifolds it is of interest to characterize the maps between open sublocales of formal Euclidean spaces

$$
\left(\mathcal{R}^{m}\right)_{\mid U} \longrightarrow\left(\mathcal{R}^{n}\right)_{\mid V} .
$$

We consider a more general version of this problem where the Euclidean spaces have been replaced by localic versions of LCM spaces. In this paper we study the correspondence between maps and morphisms when the localic completions are restricted to open sublocales

$$
M(X)_{\mid U} \longrightarrow M(Y)_{\mid V}
$$

This correspondence is not trivial from a constructive point of view. As shown in [5] the maps $M(X) \longrightarrow M(\mathbb{R})_{\mid(0, \infty)}$ correspond to continuous functions $X \longrightarrow \mathbb{R}$ that on each open ball has a positive uniform lower bound, rather than positive functions. Constructively, there is a distinction: Specker [7] gives a recursive example of a continuous positive function $[0,1] \longrightarrow \mathbb{R}$ that has no uniform positive lower bound. These considerations make it clear that the set of maps $U_{*} \longrightarrow V_{*}$ between open subspaces of LCM spaces has to meet some extra conditions to be in 1-1 correspondence to maps in (1). In Section 2 we introduce and study the appropriate categories of metric spaces, called OLCM and FLCM. In Section 3 the open sublocales of $M(X)$ are studied. Section 4 establishes full and faithful functors OLCM $\longrightarrow$ FLCM

$\longrightarrow$ FTop. The whole development is constructive in the sense of Bishop [1] and may be formalized within constructive set theory CZF with dependent choice, or in Martin-Löf type theory.

\section{Open subspaces of LCM spaces}

Bishop and Bridges [1] define a metric space $X$ to be locally compact if it is inhabited and every bounded subspace is contained in a compact subspace. It follows that such a space $X$ is complete (and separable). Below we define a category OLCM of open subspaces of locally compact metric (LCM) spaces. It is partly suggested by Definition 2.2.4 of [1], but its enunciation appears to be new.

The category of open subspaces of LCM spaces is given as follows. The objects are pairs $(X, U)$ where $X=(X, d)$ is a LCM space and $U$ is an open subset of $X$. A 
continuous map $f:(X, U) \longrightarrow(Y, V)$ between two objects is a function $f: U \longrightarrow V$ such that for any compact subset $K \Subset U$

(a) $f$ is uniformly continuous on $K$,

(b) $f[K] \Subset V$.

Here $K \Subset U$ means that $K_{r} \subseteq U$ for some $r>0$, where

$$
K_{r}=\{x \in X: d(x, K) \leq r\} .
$$

The distance $d(x, S)$ is well-defined whenever the set $S$ is located and in particular when it is totally bounded. For $S \subseteq X$, let $\bar{S}$ be the closure of $S$ in $X$, that is, the set of points in $X$ that are limits of points in $S$. We have for located $S \subseteq X$ :

$$
S \Subset U \Longleftrightarrow \bar{S} \Subset U .
$$

Moreover if $S$ is totally bounded, then $\bar{S}$ is compact. Note that $f[S]$ is totally bounded whenever $S$ is. Thus $\overline{f[K]}$ is compact, if $K$ is compact. It follows that continuous maps are closed under composition, and form a category which we shall call OLCM.

Remark 2.1 The category of locally compact metric spaces LCM may be regarded as a full subcategory of this category, given by the objects of the form $(X, X)$ since the relation $S \Subset X$ is trivially true for any compact $S \subseteq X$.

The reciprocal map $(\cdot)^{-1}:\left(\mathbb{R}, \mathbb{R}_{\neq 0}\right) \longrightarrow(\mathbb{R}, \mathbb{R})$ is continuous: suppose $K \Subset \mathbb{R}_{\neq 0}$ is compact. Thus there is $r>0$ so that

$$
d(x, K) \leq r \Longrightarrow x \neq 0 .
$$

We have $K \subseteq(-\infty,-r] \cup[r, \infty)$. Indeed, suppose $y \in K$. Then by (2) $y \neq 0$, i.e. $y<0$ or $0<y$. Consider the case $y<0$. Then $-r<y$ is impossible, since this together $y \in K$ would imply that $d(0, K)<r$ which by (2) gives $0 \neq 0$, an absurdity. Hence $y \leq-r$, i.e. $y \in(-\infty,-r]$. The case $0<y$, similarly implies $y \in[r, \infty)$. The reciprocal map is uniformly continuous on $(-\infty,-r] \cup[r, \infty)$ and, a fortiori, on $K$. Thus (a) is valid and (b) is trivially true.

A continuous map $f:[0,1] \longrightarrow\left(\mathbb{R}, \mathbb{R}_{\neq 0}\right)$ is in particular required to satisfy $f([0,1]) \Subset \mathbb{R}_{\neq 0}$. Thus $f([0,1]) \subseteq(-\infty,-r] \cup[r, \infty)$ for some $r>0$. In the recursive setting, this excludes the familiar counterexamples of Specker [7] and Julian and Richman [3] of a positive (uniformly) continuous function on [0,1] which has no positive uniform lower bound. 
Next we prepare for the definition of a still wider category, FLCM, and for the definition of the localic completion. In a metric space $X=(X, d)$ a formal open ball is a symbol $\mathrm{b}(x, \delta)$, where $x \in X$ and $\delta$ is a positive rational number. These symbols are ordered by formal inclusion $\leq$

$$
\mathrm{b}(x, \delta) \leq \mathrm{b}(y, \varepsilon) \Longleftrightarrow d(x, y)+\delta \leq \varepsilon .
$$

Replacing $\leq$ by $<$ everywhere gives the corresponding definition of strict formal inclusion $(<)$. The strict inclusion relation is extended to sets of symbols by saying that $U<V$ holds if, and only if, for each $a \in U$ there is $b \in V$ with $a<b$. The radius of a formal ball is $\rho(\mathrm{b}(x, \delta))=\delta$. Each formal open ball represents a real open ball

$$
\mathrm{b}(x, \delta)_{*}=B(x, \delta)=\{y \in X: d(x, y)<\delta\} .
$$

This representation is of course not unique in general - consider $X=[0,1]$ and $\delta>1$. For a set $N$ of formal open balls, let $N_{*}=\bigcup\left\{b_{*}: b \in N\right\}$.

Lemma 2.2 Let $X$ be a locally compact metric space. For any formal balls $a<b$ of $X$, there is a compact subset $K \subseteq X$ with

$$
a_{*} \subseteq K \subseteq b_{*} .
$$

Proof Suppose $a<b$ are formal open balls where $a=\mathrm{b}(x, \delta)$. Then there is $\varepsilon>\delta$ with $\mathrm{b}(x, \varepsilon)<b$. It now suffices to find a compact $K \subseteq X$ with $B(x, \delta) \subseteq K \subseteq B(x, \varepsilon)$. Since $X$ is locally compact there is a compact $L \supseteq B(x, \varepsilon)$. For $n \geq 1$, we let

$$
N_{n}=\left\{x_{n, 1}, \ldots, x_{n, m_{n}}\right\}
$$

be a $2^{-n}$-approximation for $L$ ([1, Def. 4.4.1]). Pick $\alpha, \beta$ with $\delta<\alpha<\beta<\varepsilon$. Then for each $i$ we have $d\left(x, x_{n, i}\right)<\beta$ or $d\left(x, x_{n, i}\right)>\alpha$. Construct by induction a function $\lambda_{n}:\left\{1, \ldots, m_{n}\right\} \longrightarrow\{0,1\}$ so that

(i) $\lambda_{n}(i)=1$ implies $d\left(x, x_{n, i}\right)<\beta$

(ii) $\lambda_{n}(i)=0$ implies $d\left(x, x_{n, i}\right)>\alpha$.

Let $N=\left\{x_{n, i}: n \geq 1, \lambda_{n}(i)=1\right\}$ and let $K$ be the set of limit points of this set. Note that we use the axiom of countable choice to construct $\lambda$. By definition $N \subseteq B(x, \beta)$ and hence $K \subseteq B(x, \varepsilon)$. Suppose now $z \in B(x, \delta)$ and $\rho>0$. Let $n \geq 1$ be large enough that $2^{-n} \leq \min (\alpha-\delta, \rho)$. Then since $z \in L$ there is some index $i \in\left\{1, \ldots, m_{n}\right\}$ with $d\left(z, x_{n, i}\right)<2^{-n}$. Then

$$
d\left(x, x_{n, i}\right) \leq d(x, z)+d\left(z, x_{n, i}\right)<\delta+\alpha-\delta=\alpha .
$$

Hence we must have $\lambda_{n}(i)=1$, so $x_{n, i} \in N$. Since $\rho>0$, this shows that $B(x, \delta) \subseteq$ $K$. 
We may characterize the relation $\Subset$ in terms of formal inclusion.

Lemma 2.3 Let $X$ be a metric space. For a totally bounded $S \subseteq X$ and open set $U \subseteq X: S \Subset U$ if, and only if, there are formal open balls in $X$

$$
b_{i}<c_{i} \quad(i=1, \ldots n),
$$

with $S \subseteq\left\{b_{1}, \ldots, b_{n}\right\}_{*}$ and $\left\{c_{1}, \ldots, c_{n}\right\}_{*} \subseteq U$.

Proof $(\Rightarrow)$ : Suppose that $S_{r} \subseteq U$ for some $r>0$. By total boundedness there is a $r / 2$-approximation $x_{1}, \ldots, x_{n}$ of $S$. We may then take $b_{i}=\mathrm{b}\left(x_{i}, r / 2\right)$ and $c_{i}=\mathrm{b}\left(x_{i}, r\right)$. We have $\left(c_{i}\right)_{*}=B\left(x_{i}, r\right) \subseteq U$, since if $d\left(y, x_{i}\right)<r$, then $d(y, S) \leq r$ and so $y \in U$.

$(\Leftarrow)$ : Suppose that $b_{i}=\mathrm{b}\left(x_{i}, \delta_{i}\right)$ and $c_{i}=\mathrm{b}\left(y_{i}, \varepsilon_{i}\right)$, where $b_{i}<c_{i}$ and $S \subseteq$ $\left\{b_{1}, \ldots, b_{n}\right\}_{*}$ and $\left\{c_{1}, \ldots, c_{n}\right\}_{*} \subseteq U$. Let $t>0$ be so small that

$$
d\left(x_{i}, y_{i}\right)+\delta_{i}+2 t<\varepsilon_{i}
$$

for all $i=1, \ldots, n$. Then we have $S_{t} \subseteq U$ : Suppose $z \in S_{t}$. Thus in particular $d(z, u) \leq 2 t$ for some $u \in S$. Hence $u \in\left(b_{i}\right)_{*}$ for some $i$, and

$$
\begin{aligned}
d\left(z, y_{i}\right) & \leq d(z, u)+d\left(u, x_{i}\right)+d\left(x_{i}, y_{i}\right) \\
& \leq 2 t+\delta_{i}+d\left(x_{i}, y_{i}\right)<\varepsilon_{i}
\end{aligned}
$$

Thus $z \in\left(c_{i}\right)_{*} \subseteq U$.

Motivated by this lemma we define yet another inclusion relation $<_{*}$. Let $X$ be a metric space. For a subset $S \subseteq X$ and a set $N$ of formal open balls of $X$ define $S<_{*} N$ to hold if and only if there is a finitely enumerable (f.e.) set $F$ of formal balls with $S \subseteq F_{*}$ and $F<N$. We pronounce $S<_{*} N$ as $S$ is formally well-included in $M$. For any open $U \subseteq X$ define $H(U)=\{\mathrm{b}(x, \delta): B(x, \delta) \subseteq U\}$. We have by Lemma 2.3 the following:

Corollary 2.4 Let $X$ be a metric space. For $S \subseteq X$ totally bounded and $U \subseteq X$ open that

$$
S \Subset U \Longleftrightarrow S<_{*} H(U) .
$$

Moreover for any set of formal open balls $N$ of $X$ and for any totally bounded $S \subseteq X$ we have

$$
S<_{*} N \Longrightarrow S \Subset N_{*} .
$$


Remark The converse of (3) implies that $[0,1]$ is point-wise covering compact, which is not provable constructively. Consider $X=S=[0,1]$ and let $N$ be a set of formal balls so that $N_{*}=X$. Clearly $S \Subset N_{*}$, and hence by the converse of (3) $S \subseteq F_{*}$ for some f.e. set $F<N$. Hence there is a f.e. set $G \subseteq N$ with $[0,1] \subseteq G_{*}$. Thus [0,1] is point-wise covering compact, and we have a effective counterexample to the reversal of the implication in (3).

\subsection{Metric complements of located subsets}

The function $H$ is part of a Galois connection: for any set $A$ of formal balls of $X$ and any open $U \subseteq X$,

$$
A_{*} \subseteq U \Longleftrightarrow A \subseteq H(U) .
$$

Lemma $2.5 H(U)_{*}=U$.

Proof It is immediate that $H(U)_{*} \subseteq U$. Moreover if $x \in U$, then there is a positive rational $\delta$ so that $B(x, \delta) \subseteq U$ thus $\mathrm{b}(x, \delta) \in H(U)$ and so $x \in H(U)_{*}$. Hence also $H(U)_{*}=U$.

It is direct from the Galois connection that $A \subseteq H\left(A_{*}\right)$. The reverse inclusion does not always hold. A set of formal balls $A$ of $X$ is point-wise saturated if $H\left(A_{*}\right)=A$. Any such set is saturated as well (see Section 3 for definition). By Lemma 2.5 it is clear that $A$ is point-wise saturated if, and only if, $A=H(U)$ for some open $U \subseteq X$.

Lemma 2.6 Let $X$ be a metric space, let $A$ be a set of formal balls of $X$ and let $S \subseteq X$ be totally bounded. If $A$ is point-wise saturated, then

$$
S<_{*} A \Longleftrightarrow S \Subset A_{*} .
$$

Proof Suppose $A=H\left(A_{*}\right)$. Then by Lemma 2.4

$$
S \Subset A_{*} \Longleftrightarrow S<_{*} H\left(A_{*}\right) \Longleftrightarrow S<_{*} A .
$$

An important class of point-wise saturated sets arise in the following way. Let $X$ be a metric space. Let $A \subseteq X$ be a located subset, and denote its metric complement by

$$
C_{A}=\{x \in X \mid d(x, A)>0\} .
$$

The formal counterpart to $C_{A}$ is

$$
S_{A}={ }_{\text {def }}\{\mathrm{b}(x, \delta): x \in X, d(x, A) \geq \delta\} .
$$

It is follows from the theorem below that $S_{A}$ is point-wise saturated. 
Example 2.7 $C_{(-\infty, 0]}=\{x \in \mathbb{R}: x>0\}$ and $S_{(-\infty, 0]}=\{\mathrm{b}(x, \delta): x \in \mathbb{R}, x \geq$ $\delta\}$.

Theorem 2.8 For any located subset $A$ of a metric space $X, C_{A}$ is an open set and

$$
H\left(C_{A}\right)=S_{A} \quad\left(S_{A}\right)_{*}=C_{A} .
$$

Proof $C_{A}$ is open: if $x \in C_{A}$, then $d(x, A)>\delta$ for some $\delta>0$. For $y \in B(x, \delta / 2)$, we have either $d(y, A)>0$ or $d(y, A)<\delta / 2$. The latter is impossible, since it would imply that there is $z \in A$ with $d(y, z)<\delta / 2$, and thus $d(x, z)<\delta$, which contradicts $d(x, A)>\delta$. Hence $B(x, \delta / 2) \subseteq C_{A}$.

$H\left(C_{A}\right)=S_{A}$ : First suppose $\mathrm{b}(x, \delta) \in H\left(C_{A}\right)$. Suppose that $d(x, A)<\delta$. Thus $d(x, y)<\delta$ for some $y \in A$. Thus $y \in H\left(C_{A}\right)$ by the assumption. Thereby $d(y, A)>0$, which is impossible since $y \in A$. Hence $d(x, A)<\delta$ is false, i.e. $d(x, A) \geq \delta$. This means that $\mathrm{b}(x, \delta) \in S_{A}$. Conversely, assume $\mathrm{b}(x, \delta) \in S_{A}$, i.e. $d(x, A) \geq \delta$. We show $B(x, \delta) \subseteq C_{A}$. Take $y$ with $d(y, x)<\delta$ and $\varepsilon$ such that $d(y, x)+\varepsilon<\delta$. Then either $d(y, A)>0$ or $d(y, A)<\varepsilon$. The latter case is actually impossible, since then there would be $z \in A$ with $d(y, z)<\varepsilon$ and so

$$
d(x, z) \leq d(y, x)+d(y, z)<d(y, x)+\varepsilon<\delta .
$$

This contradicts the assumption $d(x, A) \geq \delta$. Hence only the case $d(y, A)>0$ remains possible, i.e. $y \in C_{A}$.

$\left(S_{A}\right)_{*}=C_{A}$ : By the first equation we have $H\left(C_{A}\right)_{*}=\left(S_{A}\right)_{*}$. Now Lemma 2.5 gives $C_{A}=H\left(C_{A}\right)_{*}$.

\subsection{The Category FLCM}

We use the relation $<_{*}$ to define a new category FLCM. An object $(X, P)$ is a locally compact metric space $X$ together with a set $P$ of formal balls of $X$. A morphism $f:(X, P) \longrightarrow(Y, Q)$ between two such objects is function $f: P_{*} \longrightarrow Q_{*}$ so that for any $a<P$ we have

(a) $f: a_{*} \longrightarrow Q_{*}$ is uniformly continuous,

(b) $f\left[a_{*}\right]<_{*} Q$.

\section{Lemma 2.9 FLCM is a category.}


Proof The identity function $\operatorname{id}_{P_{*}}$ is uniformly continuous on any $a_{*}$ with $a<P$. If $a<P$, then clearly $a_{*}<_{*} P$. Hence $\operatorname{id}_{P_{*}}$ is a morphism on $(X, P)$. It now suffices to show that the morphisms are closed under composition. Suppose $f:(X, P) \longrightarrow(Y, Q)$ and $g:(Y, Q) \longrightarrow(Z, R)$ are morphisms. Let $a<P$ be some open ball. We have by (b) for $f$ that there is a f.e. set of formal open balls $F=\left\{b_{1}, \ldots, b_{n}\right\}$ with $f\left[a_{*}\right] \subseteq F_{*}$ and $F<Q$. Now

$$
(g \circ f)\left[a_{*}\right]=g\left[f\left[a_{*}\right]\right] \subseteq g\left[\left(b_{1}\right)_{*}\right] \cup \cdots \cup g\left[\left(b_{n}\right)_{*}\right] .
$$

By (b) for $g$ we get, for each $i=1, \ldots, n$, a f.e. set $G_{i}$ with $g\left[\left(b_{i}\right)_{*}\right] \subseteq\left(G_{i}\right)_{*}$ and $G_{i}<R$. Thus $(g \circ f)\left[a_{*}\right]<_{*} R$ witnessed by the f.e. set $G=G_{1} \cup \cdots \cup G_{n}$. Hence $g \circ f$ satisfies (b).

Notice also that $g$ is uniformly continuous on each $\left(b_{i}\right)_{*}$. Hence it is uniformly continuous on the f.e. union $F_{*}$ and therefore also on $f\left[a_{*}\right]$. Thus $g \circ f$ satisfies (a) as well.

Theorem 2.10 Let $X$ and $Y$ be $L C M$ spaces. Let $U \subseteq X$ and $V \subseteq Y$ be open subsets. Then $f:(X, U) \longrightarrow(Y, V)$ is continuous in OLCM if, and only if, $f:(X, H(U))$ $\longrightarrow(Y, H(V))$ is a morphism in FLCM.

Proof $(\Rightarrow)$ Suppose that $f:(X, U) \longrightarrow(Y, V)$ is continuous. Let $a<H(U)$. Thus there is a formal ball $c>a$ with $c_{*} \subseteq U$. Take a formal ball $b$ with $a<b<c$. By Lemma 2.2 there is a compact $K \subseteq X$ with $a_{*} \subseteq K \subseteq b_{*}$. Lemma 2.3 then yields $K \Subset U$. By assumption $f$ is uniformly continuous on $K$ and hence also on $a_{*}$. This verifies condition (a). We have moreover $f[K] \Subset V$, and since $f[K]$ is totally bounded, there are by Lemma 2.3 formal balls $b_{i}<c_{i}(i=1, \ldots, n)$ with $f[K] \subseteq\left\{b_{1}, \ldots, b_{n}\right\}_{*}$ and $\left\{c_{1}, \ldots, c_{n}\right\}_{*} \subseteq V$. But this says that $f[K]<_{*} V$. Now $f\left[a_{*}\right] \subseteq f[K]$ so (b) is verified.

$(\Leftarrow)$ Suppose now that $f:(X, H(U)) \longrightarrow(Y, H(V))$ is a morphism in FLCM. Let $K$ be a compact subset of $X$ with $K \Subset U$. By Lemma 2.3, we find formal balls $b_{i}<c_{i}$ $(i=1, \ldots, n)$ with $K \subseteq\left\{b_{1}, \ldots, b_{n}\right\}_{*}$ and $\left\{c_{1}, \ldots, c_{n}\right\}_{*} \subseteq U$. Thus $b_{i}<H(U)$ for each $i$. By assumption, $f$ is uniformly continuous on each $\left(b_{i}\right)_{*}$. Hence $f$ is uniformly continuous on $\left\{b_{1}, \ldots, b_{n}\right\}_{*}$, since $n$ is finite. Thus $f$ is uniformly continuous on $K$. By the assumption we have moreover that $f\left[\left(b_{i}\right)_{*}\right]<_{*} H(V)$ for each $i=1, \ldots, n$. Thus there are f.e. sets of formal balls $F^{i}<G^{i}$ with $f\left[\left(b_{i}\right)_{*}\right] \subseteq\left(F^{i}\right)_{*}$ and $\left(G^{i}\right)_{*} \subseteq V$. Thus $f[K]_{*} \subseteq\left(\cup_{i=1}^{n} F_{i}\right)_{*}$ and $\cup_{i=1}^{n} F^{i}<\cup_{i=1}^{n} G^{i}$ both f.e. and $\left(\cup_{i=1}^{n} G^{i}\right)_{*} \subseteq V$. This shows that $f[K] \Subset V$. Hence $f:(X, U) \longrightarrow(Y, V)$ is continuous. 
Corollary 2.11 The category OLCM is a full subcategory of FLCM via the embed$\operatorname{ding}(X, U) \mapsto(X, H(U))$.

Proof This follows by Theorem 2.10 and Lemma 2.5. The latter gives injectivity on objects of the categories.

The structure of formal neighbourhoods $P$ in the space $(X, P)$ is essential. One might think that only the extent of the points matter, i.e. that for sets $P$ and $Q$ of formal balls of $X$, where $X$ is a compact metric space,

$$
P_{*}=Q_{*} \Longrightarrow(X, P) \cong(X, Q) .
$$

This is not so constructively. In fact, a special case of this implies that every compact metric space is covering compact, which is constructively false. See Proposition 4.6 below.

However, if $P$ and $Q$ are point-wise saturated and $P_{*}=Q_{*}$, then obviously $P=Q$.

\section{Localic completion}

Vickers [8,9] gives a construction $M(X)$ of a formal topology from a metric space $X$ - the localic completion of $X-$ so that the canonical map $j_{X}: X \longrightarrow \operatorname{Pt}(M(X))$ is a metric completion of $X$. In particular $j_{X}$ is a metric isomorphism, in case $X$ is already complete.

The fundamentals of formal topology can be found in Sambin [6]. Here the standard notion of formal topology based on preorders is employed. Basically it is a Grothendieck topology on a preorder instead of a category. A formal topology $\mathcal{X}$ is a triple $(X, \triangleleft, \leq)$ where $X$ is the set of formal neighbourhoods, $\leq$ is a preorder relation on those and $\triangleleft$ is the formal cover relation, which is assumed to be set-presented. The relation $\triangleleft$ is defined between elements of $X$ and subsets of $X$ and is to satisfy the properties

(Refl) $a \in U$ implies $a \triangleleft U$,

(Tra) $a \triangleleft U$ and $U \triangleleft V$ implies $a \triangleleft V$,

(Loc) $a \triangleleft U$ and $a \triangleleft V$ implies $a \triangleleft U \wedge V$,

(Ext) $a \leq b$ implies $a \triangleleft\{b\}$. 
Here $U \triangleleft V$ means that $a \triangleleft V$ for each $a \in U$, and $U \wedge V$ is defined to be $\{x \in X$ : $(\exists y \in U) x \leq y \&(\exists z \in V) x \leq z\}$. A subset $U \subseteq X$ is saturated if $a \in U$ whenever $a \triangleleft U$. The saturated subsets ordered by inclusion make up the locale that the formal topology $X$ represents.

A continuous morphism between formal topologies $F: \mathcal{X} \longrightarrow \mathcal{Y}$ is a relation $R \subseteq X \times Y$, which is intended to be an abstraction and a constructive version of the relation $U \subseteq f^{-1}[V]$ for a continuous function $f$. The required properties for a continuous morphism are the following

(A1) $a F b, b \triangleleft V$ implies $a \triangleleft F^{-1} V$,

(A2) $a \triangleleft U,(\forall x \in U) x F b$ implies $a F b$,

(A3) $X \triangleleft F^{-1} Y$,

(A4) $a F b, a F c$ implies $a \triangleleft F^{-1}[b \wedge c]$.

Here $F^{-1} V$ is the subset $\{x \in X:(\exists y \in V) x F y\}$. Moreover we have written $b \wedge c$ for $\{b\} \wedge\{c\}$.

We shall here use the framework of [4]. There it was shown that the localic completion $M: \mathbf{L C M} \longrightarrow$ FTop can be made into a full and faithful functor from the category of locally compact metric spaces to the category of formal topologies. Another result of [4] is that the inductively generated cover relation $\triangleleft$ of $M(X)=(M, \triangleleft, \leq)$ may be characterized by an explicitly defined relation $\lessdot$ :

$$
a \triangleleft U \Longleftrightarrow a \lessdot U .
$$

This relation is defined as follows

$$
a \lessdot U \Longleftrightarrow(\forall b, c \in M)\left[b<c<a \Rightarrow\left(\exists U_{0} \in A(b, c)\right) U_{0}<U\right] .
$$

We explain the notation. The formal neighbourhoods of $M$ are the open formal balls $\mathrm{b}(x, \delta)$ of $X$ and are ordered as described in the previous section. The set $A(b, c)$ consists of finitely enumerable sets $C \subseteq M$ such that

$$
b \sqsubseteq C<c .
$$

The relation $b \sqsubseteq C$ says that there is a number $\delta>0$ (a "Lebesgue number") so that $b{ }_{\delta} C$. Now $b{ }_{\delta} C$ is defined to mean that any formal ball of smaller radius than $\delta$, which is included in $b$, is also included in some $q \in C$.

We also recall that the cover relation $M(X)$ is the smallest cover relation $\triangleleft$ satisfying, for all $p \in M$

(M1) $p \triangleleft\{q \in M: q<p\}$, 
(M2)

$p \triangleleft\{\mathrm{b}(x, \varepsilon): x \in X\}$, for each rational $\varepsilon>0$.

Notice that from (M2) follows by localization that

$$
V \triangleleft V^{(\varepsilon)}=V \wedge\{\mathrm{b}(x, \varepsilon): x \in X\} .
$$

All balls in $V^{(\varepsilon)}$ have radius at most $\varepsilon$.

Lemma 3.1 Let $X$ be a $L C M$ space. Then for $S \subseteq X$ and $U, V \subseteq M(X)$

$$
S<_{*} U \triangleleft V \Longrightarrow S<_{*} V .
$$

Proof Suppose $S<_{*} U \triangleleft V$. Then there is $F=\left\{b_{1}, \ldots, b_{n}\right\} \subseteq M(X)$ with $S_{*} \subseteq F_{*}$ and $F<U$. We find for each index $i=1, \ldots, n$, some $a_{i} \in U$ with $b_{i}<a_{i}$. Pick $c_{i}$ with $b_{i}<c_{i}<a_{i}$. Now $a_{i} \triangleleft V$, so $a_{i} \lessdot V$. Hence there is a f.e. $G_{i} \in A\left(b_{i}, c_{i}\right)$ with $G_{i}<V$. Thus $G=G_{1} \cup \cdots \cup G_{n}$ is f.e. and $S_{*} \subseteq G_{*}$ and $G<V$. That is $S<_{*} V$.

Two sets of neighbourhoods $U$ and $U^{\prime}$ are said to be equivalent ( $U \sim U^{\prime}$ ) if $U \triangleleft U^{\prime}$ and $U^{\prime} \triangleleft U$.

Lemma 3.2 Let $X$ and $Y$ be LCM spaces. Let $U, U^{\prime} \subseteq M(X)$ and $V, V^{\prime} \subseteq M(Y)$ and suppose that $U \sim U^{\prime}$ and $V \sim V^{\prime}$. Then $f:(X, U) \longrightarrow(Y, V)$ continuous implies that $f:\left(X, U^{\prime}\right) \longrightarrow\left(Y, V^{\prime}\right)$ is continuous.

Proof Suppose $f:(X, U) \longrightarrow(Y, V)$ continuous. Consider an arbitrary $p<U^{\prime}$. Then there is $a \in U^{\prime}$ and $q$ with $p<q<a$. As $U \sim U^{\prime}$, we have $a \triangleleft U$. Hence also $a \lessdot U$. Thus there is a f.e. $F=\left\{p_{1}, \ldots, p_{n}\right\} \in A(p, q)$ with $F<U$. By assumption $f$ is uniformly continuous on each $\left(p_{i}\right)_{*}$. Hence $f$ is uniformly continuous also on $p_{*} \subseteq F_{*}$. Moreover by assumption, $f\left[\left(p_{i}\right)_{*}\right]<_{*} V$. It follows that $f\left[F_{*}\right]<_{*} V$. By Lemma 3.1 and $V \triangleleft V^{\prime}$, we get $f\left[F_{*}\right]<_{*} V^{\prime}$. Since $f\left[p_{*}\right] \subseteq f\left[F_{*}\right]$ we get $f\left[p_{*}\right]<_{*} V^{\prime}$. Thereby we have proved that $f:\left(X, U^{\prime}\right) \longrightarrow\left(Y, V^{\prime}\right)$ is continuous.

Corollary 3.3 Let $X$ be a $L C M$ space and let $U \subseteq M(X)$ be a set of formal balls. Then the identity function $i$ on $U_{*}$ gives an isomorphism

$$
i:(X, U) \longrightarrow(X, \tilde{U})
$$

in FLCM. Here $\tilde{U}=\{a \in M(X): a \triangleleft U\}$, the saturation of $U$.

By this corollary it is enough to consider objects $(X, U)$ in FLCM where $U \subseteq M(X)$ is a saturated subset with respect to the cover relation of $M(X)$. Let SLCM denote the full subcategory of FLCM determined by such objects. 
Corollary 3.4 The functor $J:$ FLCM $\longrightarrow$ SLCM given by $J(X, U)=(X, \tilde{U})$ and $J(f)=f$ and the inclusion functor in the reverse direction, form an equivalence of categories. In particular we have that $J:$ FLCM $\longrightarrow$ SLCM is full and faithful.

Proof This follows from Corollary 3.3 and Lemma 3.2.

\subsection{Open sublocales}

Let $X=(X, \triangleleft, \leq)$ be a formal topology, and let $G \subseteq X$ be a set of neighbourhoods. Then the open subspace $X_{\mid G}$ of $X$ determined by $G$ is defined as follows. Let $X_{\mid G}=$ $\left(X, \triangleleft^{\prime}, \leq\right)$, which is as $X$ except that we change the cover relation $\triangleleft$ of $X$ to be the one defined by

$$
a \triangleleft^{\prime} U \Longleftrightarrow \text { def } a \wedge G \triangleleft U .
$$

Note that $U_{1} \triangleleft^{\prime} U_{2}$ iff $U_{1} \wedge G \triangleleft U_{2} \wedge G$. Hence only the parts covered by $G$ counts when comparing two open sets. Also if $G \sim G^{\prime}$ then

$$
a \wedge G \triangleleft U \Longleftrightarrow a \wedge G^{\prime} \triangleleft U .
$$

It is thus sufficient to consider $G$ s that are saturated subsets of $X$. Such subsets are necessarily down-closed, i.e. $G_{\leq}=G$.

We have the following useful results about regular formal topologies. Recall that a formal topology $X=(X, \leq, \triangleleft)$ is regular if for all $b \in X$,

$$
b \triangleleft \mathrm{wc}(b)
$$

where $\operatorname{wc}(b)=\{a \in X: a \lll b\}$ and

$$
a \lll b \Longleftrightarrow \text { def } X \triangleleft a^{\perp} \cup\{b\},
$$

and moreover $a^{\perp}=\{u \in X: u \wedge a \triangleleft \emptyset\}$.

Lemma 3.5 Let $X$ be a formal topology and let $G \subseteq X$ be an arbitrary set of neighbourhoods. If $X$ is regular, then so is $X_{\mid G}$.

Proof Suppose that $X=(X, \leq, \triangleleft)$ is regular. We prove that $X_{\mid G}=(X, \leq, \triangleleft)$ is regular. Now let $a^{\perp^{\prime}}, \lll^{\prime}$ and $\mathrm{wc}^{\prime}(b)$ be the terms for $X_{\mid G}$ involved in the definition of regularity. We wish to prove $b \triangleleft^{\prime} \mathrm{wc}^{\prime}(b)$, i.e. that for an arbitrary $d \leq b$ with $d \leq G$ we have $d \triangleleft \mathrm{wc}^{\prime}(b)$. By regularity of $X: d \triangleleft \mathrm{wc}(d)$. Now wc $(d) \subseteq \mathrm{wc}^{\prime}(b)$, as for any $a$ :

$$
a^{\perp} \cup\{d\} \triangleleft a^{\perp^{\prime}} \cup\{b\} .
$$

Thus $d \triangleleft \mathrm{wc}(d) \subseteq \mathrm{wc}^{\prime}(b)$. 
Lemma 3.6 Suppose that $F, G: X \longrightarrow Y$ are continuous morphisms between formal topologies, where $Y$ is regular. If $F \subseteq G$, then $F=G$.

Proof Suppose that $F \subseteq G$. Since $Y$ is regular we have for any $b$ that $G^{-1} b \triangleleft G^{-1}[\operatorname{wc}(b)]$. To prove $G \subseteq F$ it will thus be enough to prove

$$
G^{-1}[\mathrm{wc}(b)] \triangleleft F^{-1} b .
$$

Suppose that $a G c$ with $c \lll b$. Thus $Y \triangleleft c^{\perp} \cup\{b\}$. Hence by (A3) for $F$ and localization we obtain

$$
a \triangleleft F^{-1}\left[c^{\perp} \cup\{b\}\right] \wedge a .
$$

To prove $a \triangleleft F^{-1} b$ it is enough to show

$$
F^{-1}\left[c^{\perp} \cup\{b\}\right] \wedge a \triangleleft F^{-1} b .
$$

Suppose that $u \leq a, u F d$ where $d \in c^{\perp} \cup\{b\}$. In case $d=b, u \in F^{-1} b$ and so $u \triangleleft F^{-1} b$ is clear. In case $d \in c^{\perp}$, it holds that $d \wedge c \triangleleft \emptyset$. Now $u F d$ implies $u G d$ by assumption and as $u \leq a$ we gave also $u G c$. Hence

$$
u \triangleleft G^{-1}[d \wedge c] \triangleleft G^{-1}[\emptyset] \triangleleft \emptyset .
$$

Thus, trivially, also $u \triangleleft F^{-1} b$.

Lemma 3.7 Let $X$ and $Y$ be formal topologies and let $U \subseteq X$ and $V \subseteq Y$ be saturated subsets. Suppose that $F, G: X_{\mid U} \longrightarrow Y_{\mid V}$ are continuous mappings with $F \cap(U \times V) \subseteq G$. Then $F \subseteq G$.

Proof Suppose that $a F b$, with $a \in X$ and $b \in Y$ arbitrary. Then we have $b \triangleleft_{Y_{\mid V}} b \wedge V$. Hence by (A1) for $F$, and the definition of the covering relation in $M_{\mid U}$

$$
a \wedge U \triangleleft_{X} U \wedge F^{-1}[b \wedge V] .
$$

But the assumption $F \cap(U \times V) \subseteq G$ implies that

$$
U \wedge F^{-1}[b \wedge V] \subseteq U \wedge G^{-1}[b \wedge V] .
$$

Hence $a \wedge U \triangleleft_{X} U \wedge G^{-1}[b \wedge V] \triangleleft_{X} G^{-1} b$ and consequently $a G b$. 


\subsection{Open sublocales of localic completions}

Let $X$ be a complete metric space, and let $G \subseteq M(X)$ be a subset. The canonical metric isomorphism $j_{X}: X \longrightarrow \operatorname{Pt}(M(X))$ restricts to a metric isomorphism

$$
j_{X, G}=G_{*} \longrightarrow G^{*}=\operatorname{Pt}\left(M(X)_{\mid G}\right)=\operatorname{Pt}\left(M(X)_{\mid \tilde{G}}\right)
$$

i.e. $j_{X, G}(x)=\{\mathrm{b}(y, \delta) \in M(X): d(x, y)<\delta\}$. Note that

$$
b \in j(x) \Longleftrightarrow x \in b_{*} .
$$

Let $X$ be a LCM space. For a saturated subset $G \subseteq M(X)$, the open sublocale $M(X)_{\mid G}$ has the following characterization of its cover relation

$$
a \triangleleft^{\prime} U \Longleftrightarrow a \wedge G \lessdot U .
$$

Note that since $G$ is down-closed with respect to $<$ we get

$$
a \wedge G \lessdot U \Longleftrightarrow(\forall b, c \in G)\left[b<c<a \Rightarrow\left(\exists U_{0} \in A(b, c)\right) U_{0}<U\right] .
$$

Further, note that if $c \in G$ then each set in $A(b, c)$ is a subset of $G$.

Suppose that $Y$ is an arbitrary metric space and that $H \subseteq M(Y)$ is a saturated subset. For $F: M(X)_{\mid G} \longrightarrow M(Y)_{\mid H}$ the relation $a \triangleleft^{\prime} F^{-1} V$, where $a \in G$ and $V \subseteq H$, is characterized by

$$
(\forall b, c \in G)\left[b<c<a \Rightarrow\left(\exists\left\{u_{1}, \ldots, u_{n}\right\} \in A(b, c)\right)\left(\exists\left\{v_{1}, \ldots, v_{n}\right\}<V\right)(\forall i) u_{i} F v_{i}\right] .
$$

This follows since $F^{-1} V \triangleleft^{\prime} F^{-1}\{v \in M(Y): v<V\}$.

Theorem 3.8 Let $X$ be a $L C M$ space and let $G$ be a subset of $M(X)$. Let $Y$ be a complete metric space and $H$ be a subset of $M(Y)$. There are thus metric isomorphisms

$$
j_{X, G}: G_{*} \longrightarrow \operatorname{Pt}\left(M(X)_{\mid \tilde{G}}\right) \quad j_{Y, H}: H_{*} \longrightarrow \operatorname{Pt}\left(M(Y)_{\mid \tilde{H}}\right)
$$

Suppose $F: M(X)_{\mid \tilde{G}} \longrightarrow M(Y)_{\mid \tilde{H}}$ is a continuous morphism. Then $f:(X, G)$ $\longrightarrow(Y, H)$ defined as the composition $j_{Y, H}^{-1} \circ \operatorname{Pt}(F) \circ j_{X, G}$ is a continuous map in FLCM. Moreover for $a \in \tilde{G}, b \in \tilde{H}$

$$
a F b \text { implies } f\left[a_{*}\right] \subseteq b_{*} .
$$

Proof We make the abbreviations $A=M(X)_{\mid \tilde{G}}$ and $B=M(Y)_{\mid \tilde{H}}$. Let $p<G$. We prove that $f$ is uniformly continuous on $p_{*}$ : Let $\varepsilon>0$. Then by (8) we have $B \triangleleft_{B} H \triangleleft_{B} H^{(\varepsilon / 2)}$. Thus for any $a \in A$

$$
a \triangleleft_{A} F^{-1}[B] \triangleleft_{A} F^{-1}\left[H^{(\varepsilon / 2)}\right] .
$$


Now $p<G$, so there some $a \in G$ with $p<a$. Pick further $b, c$ with $p<$ $b<c<a$. By the characterization (10) we get $U=\left\{u_{1}, \ldots, u_{n}\right\} \in A(b, c)$ and $V=\left\{v_{1}, \ldots, v_{n}\right\}<H^{(\varepsilon / 2)}$ with $u_{i} F v_{i}$ for all $i=1, \ldots, n$. Since $b \sqsubseteq U<c$, there is a rational $\theta>0$ such that for all $q \in A$

$$
\rho(q) \leq \theta \text { and } q \leq b \Longrightarrow q \leq U .
$$

Write $p=\mathrm{b}(z, \alpha)$ and $b=\mathrm{b}\left(z^{\prime}, \alpha\right)$. Since $p<b$, we can find $\theta^{\prime}>0$ with

$$
d\left(z, z^{\prime}\right)+\alpha+\theta^{\prime}<\alpha^{\prime} .
$$

Then pick $\delta>0$ so that $\delta<\theta / 2, \theta^{\prime} / 2$.

To prove uniform continuity of $f$ on $p_{*}$ we show that for $x, y \in p_{*}$ :

$$
d(x, y)<\delta \Longrightarrow d(f(x), f(y)) \leq \varepsilon .
$$

Suppose $x, y \in p_{*}$ with $d(x, y)<\delta$. Put $q=\mathrm{b}(x, 2 \delta)$. Then $\rho(q)<\theta$ and $q \leq b$ by (12) and $x \in p_{*}$. Thus by (11) there is some $i$ with $q \leq u_{i}$. As $u_{i} F v_{i}$, we have a fortiori $q F v_{i}$. Now $x, y \in q_{*}$ so $f(x), f(y) \in\left(v_{i}\right)_{*}$. Hence $d(f(x), f(y)) \leq \varepsilon$ as the radius of $v_{i}$ is no greater than $\varepsilon / 2$.

We verify the second condition for a morphism of FLCM. Suppose $p<G$. Let $\varepsilon=1$ (in fact, any positive rational number would work) and let $p<b<c<a, U$ and $V$ be as obtained above. From $b \sqsubseteq U$ follows that $p_{*} \subseteq b_{*} \subseteq U_{*}$. We have $f\left[\left(u_{i}\right)_{*}\right] \subseteq\left(v_{i}\right)_{*}$ and hence $f\left[p_{*}\right] \subseteq V_{*}$. Since $V<H^{(\varepsilon / 2)} \leq H$, we have indeed $f\left[p_{*}\right]<_{*} H$.

\section{The embedding functor}

For a continuous map $f:(X, U) \longrightarrow(Y, V)$ in FLCM we define two relations $D_{f}, B_{f} \subseteq U \times V$

$$
a D_{f} b \Longleftrightarrow\left(\exists b^{\prime} \in V\right) f\left[a_{*}\right] \subseteq b_{*}^{\prime} \& b^{\prime}<b
$$

and

$$
a B_{f} b \Longleftrightarrow\left(\exists a^{\prime} \in U\right) a<a^{\prime} \& a^{\prime} D_{f} b
$$

Lemma 4.1 Let $f:(X, U) \longrightarrow(Y, V)$ be continuous map in FLCM where $U$ and $V$ are saturated sets of formal balls. Suppose that $a<b<U$ and $C, E \subseteq V$ satisfies

$$
f\left[b_{*}\right]<_{*} C \text { and } C \sqsubseteq E .
$$

Then there is $S \in A(a, b)$ with

$$
(\forall d \in S)(\exists e \in E) d B_{f} e
$$


Proof From $f\left[b_{*}\right]<_{*} C$ follows that there are formal balls $c_{1}, \ldots, c_{n} \in C$ and $c_{1}^{\prime}, \ldots, c_{n}^{\prime} \in V$ with

$$
f\left[b_{*}\right] \subseteq\left\{c_{1}^{\prime}, \ldots, c_{n}^{\prime}\right\}_{*} \quad c_{i}^{\prime}<c_{i} \quad\left\{c_{1}, \ldots, c_{n}\right\} \sqsubseteq E .
$$

Pick a rational $\gamma>0$ so small that $c_{i} \sqsubseteq_{\gamma} E$ for all $i=1, \ldots, n$. Write

$$
c_{i}^{\prime}=\mathrm{b}\left(x_{i}, \alpha_{i}\right) \quad c_{i}=\mathrm{b}\left(y_{i}, \beta_{i}\right)
$$

As $c_{i}^{\prime}<c_{i}$ we can find a rational $\theta_{i}>0$ with

$$
d\left(x_{i}, y_{i}\right)+\alpha_{i}+\theta_{i}<\beta_{i} .
$$

Then take $\varepsilon=\min \left(\theta_{1}, \ldots, \theta_{n}, \gamma\right)$. Now $f$ is uniformly continuous on $b_{*}$, so there is $\delta>0$ with

$$
\left(\forall v, w \in b_{*}\right)[d(v, w)<\delta \Longrightarrow d(f(v), f(w))<\varepsilon / 2] .
$$

Since $X$ is locally compact and $a<b$ there is a $S \in A(a, b)$ where each ball in $S$ has radius smaller than $\delta$. (See Lemma 4.7 in [4].)

Now pick any $d=\mathrm{b}(z, \sigma) \in S$. Thus $d<b$ and $z \in b_{*}$. Since $f\left[b_{*}\right] \subseteq\left\{c_{1}^{\prime}, \ldots, c_{n}^{\prime}\right\}_{*}$, we get $f(z) \in\left(c_{i}^{\prime}\right)_{*}$ for some $i=1, \ldots, n$. Thus $d\left(f(z), x_{i}\right)<\alpha_{i}$. Hence by (13)

$$
d\left(f(z), y_{i}\right)+\varepsilon \leq d\left(f(z), x_{i}\right)+d\left(x_{i}, y_{i}\right)+\varepsilon<\alpha_{i}+d\left(x_{i}, y_{i}\right)+\varepsilon<\beta_{i} .
$$

This implies that $\mathrm{b}(f(z), \varepsilon)<c_{i}$. But $\varepsilon \leq \gamma$ and since $c_{i} \sqsubseteq_{\gamma} E$, there is some $e \in E$ with

$$
\mathrm{b}(f(z), \varepsilon) \leq e .
$$

Next let $d^{\prime}=\mathrm{b}\left(z, \sigma^{\prime}\right)$ where $\sigma^{\prime}$ is sufficiently small that $\sigma<\sigma^{\prime}<\delta$ and $d<d^{\prime}<b$. We claim that

$$
f\left[d_{*}^{\prime}\right] \subseteq \mathrm{b}(f(z), \varepsilon / 2)
$$

This together with $d<d^{\prime}$ and $\mathrm{b}(f(z), \varepsilon / 2)<\mathrm{b}(f(z), \varepsilon)$ and (15) yields

$$
d B_{f} e
$$

as required. We prove (16). Let $v \in f\left[d_{*}^{\prime}\right]$. Thus $v=f(u)$ for some $u$ with $d(u, z)<\sigma^{\prime}<\delta$. By (14) we have $d(f(u), f(z))<\varepsilon / 2$. Thus $v \in \mathrm{b}(f(z), \varepsilon / 2)_{*}$.

Let $f:(X, U) \longrightarrow(Y, V)$ be a continuous map in FLCM where $U$ and $V$ are saturated sets of formal balls. We shall define a morphism of formal topologies

$$
A_{f}: M(X)_{\mid U} \longrightarrow M(Y)_{\mid V}
$$

by

$$
a A_{f} b \Longleftrightarrow_{\text {def }} a \triangleleft_{M(X)_{\mid U}} D_{f}^{-1}[b \wedge V] .
$$

The right hand side is equivalent to $a \wedge U \triangleleft_{M(X)} D_{f}^{-1}[b \wedge V]$ 
Lemma 4.2 If $f:(X, U) \longrightarrow(Y, V)$ a continuous map in FLCM, where $U$ and $V$ are saturated, then $A_{f}: M(X)_{\mid U} \longrightarrow M(Y)_{\mid V}$ is a continuous morphism between formal topologies.

Proof Let $M_{1}=M(X)_{\mid U}$ and $M_{2}=M(Y)_{\mid V}$. We verify the continuity conditions for $A=A_{f}$. Explicitly these are

(A1) $a A b$ and $b \triangleleft_{M_{2}} W \Rightarrow a \triangleleft_{M_{1}} A^{-1} W$,

(A2) $a \triangleleft_{M_{1}} W$ and $(\forall c \in W) c A b \Rightarrow a A b$,

(A3) $M_{1} \triangleleft M_{1} A^{-1}\left[M_{2}\right]$,

(A4) $a A b_{1}$ and $a A b_{2} \Rightarrow a \triangleleft_{M_{1}} A^{-1}\left[b_{1} \wedge b_{2}\right]$.

We notice that (A2) is immediate by the transitivity of $\triangleleft_{M_{1}}$. To verify the other conditions it useful to note that

$$
a \triangleleft_{M_{1}} A^{-1} W \Longleftrightarrow a \triangleleft_{M_{1}} D^{-1}[W \wedge V]
$$

where $D=D_{f}$.

Proof of (A1): Suppose $a A b$ and $b \triangleleft_{M_{2}} W$. By (17) it is sufficient to establish $a \triangleleft_{M_{1}} D^{-1}[W \wedge V]$. Since $a A b$ means $a \triangleleft_{M_{1}} D^{-1}[b \wedge V]$, it is enough to prove

$$
D^{-1}[b \wedge V] \triangleleft_{M_{1}} D^{-1}[W \wedge V] .
$$

Take $q \in D^{-1}[b \wedge V]$. By the axiom (M1) we need only to prove $q^{\prime} \triangleleft_{M_{1}} D^{-1}[W \wedge V]$ for an arbitrary $q^{\prime}<q$. Let $q^{\prime}<q$. Pick $q^{\prime \prime}$ with $q^{\prime}<q^{\prime \prime}<q$. There is $d \in b \wedge V$ with $q D d$. Hence there is $c<d$ with $f\left[q_{*}\right] \subseteq c_{*}$. Since $U$ and $V$ are saturated, they are also down-closed. Thus $q, q^{\prime} \in U$ and $c, d \in V$. Now $b \triangleleft_{M_{2}} W$ and $d \in b \wedge V$ implies $d \triangleleft_{M(Y)} W \wedge V$. By (5) we thus have $d \lessdot W \wedge V$. Pick $c^{\prime}, c^{\prime \prime}$ so that $c<c^{\prime}<c^{\prime \prime}<d$. Hence there is $E \in A\left(c^{\prime}, c^{\prime \prime}\right)$ with $E<W \wedge V$. We have $f\left[q_{*}^{\prime \prime}\right] \subseteq f\left[q_{*}\right]<_{*}\left\{c^{\prime}\right\}$ and $\left\{c^{\prime}\right\} \sqsubseteq E$. Then we can apply Lemma 4.1 and get $S \in A\left(q^{\prime}, q^{\prime \prime}\right)$ with

$$
(\forall u \in S)(\exists e \in E) u B_{f} e .
$$

Thus

$$
S \subseteq D^{-1}[W \wedge V]
$$

Since $q^{\prime} \sqsubseteq S$, we have $q^{\prime} \triangleleft_{M(X)} S$ and thus $q^{\prime} \triangleleft_{M(X)} D^{-1}[W \wedge V]$.

Proof of (A3): By (17) it is sufficient to show $M_{1} \triangleleft_{M_{1}} D^{-1}\left[M_{2} \wedge V\right]$. But since $U$ and $V$ are both down-closed, this is equivalent to verifying $U \triangleleft_{M(X)} D^{-1} V$. By (5) this is in turn equivalent to $U \lessdot D^{-1} V$. Take an arbitrary $a \in U$ and take arbitrary $b$ and $c$ with $b<c<a$. Now $c<U$, so by the continuity of $f$ there is $P=\left\{p_{1}, \ldots, p_{n}\right\}<V$ 
with $f\left[c_{*}\right] \subseteq P_{*}$. Thus we find $E=\left\{q_{1}, \ldots, q_{n}\right\} \subseteq V$ with $p_{i}<q_{i}$ and further $C=\left\{p_{1}^{\prime}, \ldots, p_{n}^{\prime}\right\}$ with $p_{i}<p_{i}^{\prime}<q_{i}$. This implies that $f\left[c_{*}\right]<_{*} C$ and $C \sqsubseteq E$. By Lemma 4.1 we get $S \in A(b, c)$ with

$$
(\forall d \in S)(\exists e \in E) d B_{f} e .
$$

But $d B_{f} e$ and $e \in E \subseteq V$ implies $d<D^{-1} V$. Hence $b \sqsubseteq S<D^{-1} V$. Thus also $b \triangleleft_{M_{1}} D^{-1} V$. Now since $a \triangleleft_{M_{1}}\{b \in U: b<a\}$ by axiom (M1) we get by transitivity $a \triangleleft_{M_{1}} D^{-1} V$. Hence $U \triangleleft_{M_{1}} D^{-1} V$.

Proof of (A4): Suppose $a A b_{1}$ and $a A b_{2}$. By (17) and localization,

$$
a \triangleleft_{M_{1}} D^{-1}\left[b_{1} \wedge V\right] \wedge D^{-1}\left[b_{2} \wedge V\right]=D^{-1}\left[b_{1} \wedge V\right] \cap D^{-1}\left[b_{2} \wedge V\right] .
$$

By (17) it is enough to show $a \triangleleft_{M_{1}} D^{-1}\left[b_{1} \wedge b_{2} \wedge V\right]$. This is done as soon as we have shown

$$
D^{-1}\left[b_{1} \wedge V\right] \cap D^{-1}\left[b_{2} \wedge V\right] \triangleleft{ }_{M(X)} D^{-1}\left[b_{1} \wedge b_{2} \wedge V\right] .
$$

Let $d$ be an arbitrary element in the set on the left hand side. Thus there are $c_{i}^{\prime}<c_{i} \in$ $b_{i} \wedge V$ with $f\left[d_{*}\right] \subseteq\left(c_{i}^{\prime}\right)_{*}$. Moreover $V$ is down-closed so $c_{i} \in V$ and $c_{i} \leq b_{i}$. Write $c_{i}=\mathrm{b}\left(z_{i}, \gamma_{i}\right)$ and $c_{i}^{\prime}=\mathrm{b}\left(z_{i}^{\prime}, \gamma_{i}^{\prime}\right)$. Pick $\varepsilon>0$ small enough that

$$
d\left(z_{i}^{\prime}, z_{i}\right)+\gamma_{i}^{\prime}+2 \varepsilon<\gamma_{i},
$$

for $i=1,2$. We wish to prove $d \triangleleft_{M(X)} D^{-1}\left[b_{1} \wedge b_{2} \wedge V\right]$. We use (5). Suppose that $d^{\prime}<d^{\prime \prime}<d$. It is enough to find $C \in A\left(d^{\prime}, d^{\prime \prime}\right)$ with $C<D^{-1}\left[b_{1} \wedge b_{2} \wedge V\right]$. Pick $p$ so that $d^{\prime \prime}<p<d$. We have $d \in U$, so $p<U$. The function $f$ is thus uniformly continuous on $p_{*}$ and we find $\delta>0$ with

$$
\left(\forall x, y \in p_{*}\right)[d(x, y)<\delta \Longrightarrow d(f(x), f(y))<\varepsilon] .
$$

Now as $X$ is locally compact there is $C=\left\{\mathrm{b}\left(u_{1}, \alpha_{1}\right), \ldots, \mathrm{b}\left(u_{n}, \alpha_{n}\right)\right\} \in A\left(d^{\prime}, d^{\prime \prime}\right)$ where $\alpha_{j}<\delta$ for all $j=1, \ldots, n$. (Lemma 4.7 of [4].) We may find a small $\beta>0$ so that $\mathrm{b}\left(u_{j}, \alpha_{j}+\beta\right)<d^{\prime \prime}$ and $\alpha_{j}+\beta<\delta$ for all $j=1, \ldots, n$. To prove $C<D^{-1}\left[b_{1} \wedge b_{2} \wedge V\right]$ it is now sufficient to demonstrate that $\mathrm{b}\left(u_{j}, \alpha_{j}+\beta\right) \in D^{-1}\left[b_{1} \wedge b_{2} \wedge V\right]$ for each $j$. Now $u_{j} \in d_{*}^{\prime \prime} \subseteq p_{*}$ and (19) gives

$$
f\left[\mathrm{~b}\left(u_{j}, \alpha_{j}+\beta\right)_{*}\right] \subseteq \mathrm{b}\left(f\left(u_{j}\right), \varepsilon\right)_{*} .
$$

Obviously $\mathrm{b}\left(f\left(u_{j}\right), \varepsilon\right)<\mathrm{b}\left(f\left(u_{j}\right), 2 \varepsilon\right)$, so if we can show $\mathrm{b}\left(f\left(u_{j}\right), 2 \varepsilon\right) \in b_{1} \wedge b_{2} \wedge V$ we are done. To do this we show that $\mathrm{b}\left(f\left(u_{j}\right), 2 \varepsilon\right)<c_{i}=\mathrm{b}\left(z_{i}, \gamma_{i}\right)$ for $i=1,2$. This is the same as proving

$$
d\left(f\left(u_{j}\right), z_{i}\right)+2 \varepsilon<\gamma_{i} .
$$


Since $f\left(u_{j}\right) \in f\left[p_{*}\right] \subseteq f\left[d_{*}\right] \subseteq c_{i}^{\prime}$ and $d\left(f\left(u_{j}\right), z_{i}^{\prime}\right) \leq \gamma_{i}^{\prime}$ we have

$$
d\left(f\left(u_{j}\right), z_{i}\right)+2 \varepsilon \leq d\left(f\left(u_{j}\right), z_{i}^{\prime}\right)+d\left(z_{i}^{\prime}, z_{i}\right)+2 \varepsilon \leq \gamma_{i}^{\prime}+d\left(z_{i}^{\prime}, z_{i}\right)+2 \varepsilon .
$$

Thus by (18) we get the desired (20).

Lemma 4.3 Let $f:(X, U) \longrightarrow(Y, V)$ be a continuous map in FLCM, where $U$ and $V$ are saturated. Then the diagram

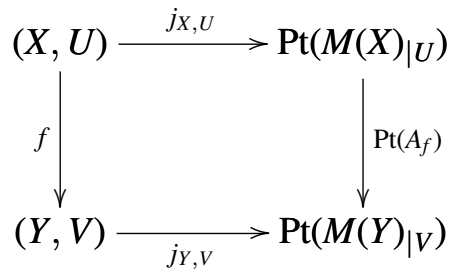

commutes.

Proof Let $x \in U_{*}$. Then using that points split covers we get

$$
\begin{aligned}
\operatorname{Pt}\left(A_{f}\right)\left(j_{X, U}(x)\right) & =\left\{b \in M(Y)_{\mid V}:\left(\exists a \in j_{X, U}(x)\right) a A_{f} b\right\} \\
& =\left\{b \in M(Y)_{\mid V}:\left(\exists a \in j_{X, U}(x)\right) a \in U \& a D_{f} b\right\} \\
& =\left\{b \in M(Y)_{\mid V}:(\exists a \in U)(\exists c \in V) x \in a_{*} \& f\left[a_{*}\right] \subseteq c_{*} \& c<b\right\}
\end{aligned}
$$

For any $b \in \operatorname{Pt}\left(A_{f}\right)\left(j_{X, U}(x)\right)$, we have $f(x) \in b_{*}$ and so $b \in j_{Y, V}(f(x))$. Conversely, suppose that $b \in j_{Y, V}(f(x))$. Thus $f(x) \in b_{*}$. Since $x \in U_{*}$, we have also $f(x) \in V_{*}$. Take $d \in V$ with $f(x) \in d_{*}$. Chose $\varepsilon>0$ so small that $q=\mathrm{b}(f(x), \varepsilon)<b$ and $q<d$. Let $\delta>0$ be so small that both $p=\mathrm{b}(x, \delta)<U$ and (by continuity of $f) f\left[p_{*}\right] \subseteq q_{*}$. Therefore $b \in \operatorname{Pt}\left(A_{f}\right)\left(j_{X, U}(x)\right)$.

Lemma 4.4 Let $X$ and $Y$ be LCM spaces. Suppose that $U \subseteq M(X)$ and $V \subseteq M(Y)$ are saturated sets. Let $F: M(X)_{\mid U} \longrightarrow M(Y)_{\mid V}$ be a continuous map. Then $F=A_{f}$ where $f:(X, U) \longrightarrow(Y, V)$ is given by

$$
f=j_{Y, V}^{-1} \circ \operatorname{Pt}(F) \circ j_{X, U} .
$$

Proof Since $M(Y)$ is a regular formal topology the open subspace $M_{2}=M(Y)_{\mid V}$ is regular too (Lemma 3.5). Thus to prove $F=A_{f}$ it is, by Lemma 3.6, sufficient to check that $F \subseteq A_{f}$. By Lemma 3.7 it is enough to verify that $F \cap(U \times V) \subseteq A_{f}$. Assume that $a F b$ where $a \in U$ and $b \in V$. By axiom (M1) $b \triangleleft_{M_{2}}\{p: p<b\}$ 
where $M_{2}=M(Y)_{\mid V}$. Thus $a \triangleleft_{M_{1}} F^{-1}\{p: p<b\}$ and hence by localization $a \triangleleft M_{1} U \wedge F^{-1}\{p: p<b\}$. We prove

$$
U \wedge F^{-1}\{p: p<b\} \subseteq A_{f}^{-1} b .
$$

If $c$ is in the left hand side, we have $c \in U$ and $c F p$ for some $p<b$. Hence by Theorem 3.8, $f\left[c_{*}\right] \subseteq p_{*}$. Thus $c D_{f} b$, and hence $c \in A_{f}^{-1} b$. Thus $a \triangleleft M_{1} A_{f}^{-1} b$. Therefore $a A_{f} b$.

Theorem 4.5 There is a full and faithful functor $M:$ SLCM $\longrightarrow$ FTop given by

$$
M(X, U)=M(X)_{\mid U} \text { and } M(f)=A_{f} .
$$

By composition

$$
M J: \text { FLCM } \longrightarrow \text { FTop }
$$

is a full and faithful functor as well. Further composition gives a fully faithful functor

$$
M J K: \text { OLCM } \longrightarrow \text { FTop, }
$$

where $K(X, U)=(X, H(U))$ and $K(f)=f$.

Proof Functoriality of $M$ : To prove this we employ the functoriality of Pt. Let $(X, U)$ be an object in SLCM. By Lemma 4.4 we have $\operatorname{id}_{M(X, U)}=A_{h}$ where

$$
h=j^{-1} \circ \operatorname{Pt}\left(\operatorname{id}_{M(X, U)}\right) \circ j=j^{-1} \circ \operatorname{id} \operatorname{Pt}(M(X, U)) \circ j=j^{-1} \circ j=\operatorname{id}_{(X, U)} .
$$

Let $g:(X, U) \longrightarrow(Y, V)$ and $f:(Y, V) \longrightarrow(Z, W)$ be continuous functions in SLCM. Using functoriality of Pt and Lemma 4.3 one obtains

$$
j^{-1} \circ \operatorname{Pt}\left(A_{f} \circ A_{g}\right) \circ j=j^{-1} \circ \operatorname{Pt}\left(A_{f}\right) \circ j^{-1} \circ j \circ \operatorname{Pt}\left(A_{g}\right) \circ j=f \circ g .
$$

By Lemma 4.4 we have $A_{f} \circ A_{g}=A_{h}$ where $h$ is the left hand side in the equation above. Thus $h=f \circ g$. This establishes functoriality of $M$.

Fullness: For any $F: M(X, U) \longrightarrow M(Y, V)$ we have by Lemma 4.4 some $f:(X, U)$ $\longrightarrow(Y, V)$ so that $F=A_{f}=M(f)$.

Faithfulness: If $M(f)=M(g)$, then $A_{f}=A_{g}$ and so by Lemma 4.3

$$
f=j^{-1} \circ \operatorname{Pt}\left(A_{f}\right) \circ j=j^{-1} \circ \operatorname{Pt}\left(A_{g}\right) \circ j=g .
$$

The functors $J:$ FLCM $\longrightarrow$ SLCM and $K:$ OLCM $\longrightarrow$ FLCM both are full and faithful according Corollary 3.4 and Corollary 2.11 respectively. 
Finally, we can prove what was stated at the end of Section 2: that the point-wise structure of the objects in FLCM is not enough, unless we assume non-constructive axioms. We have:

Proposition 4.6 Let $X$ be a compact metric space. Then $X$ is covering compact if for any $P \subseteq M(X)$,

$$
P_{*}=X \Longrightarrow(X, P) \cong(X, H(X)) \text {. }
$$

Proof Assume that the condition holds. Suppose that $P \subseteq M(X)$ and suppose that $P_{*}=X$. Then $(X, P) \cong(X, H(X))$ by the condition. Thus since $M$ is functor we have $M(X, P) \cong M(X, H(X)) \cong M(X)$. Now $M(X)$ is compact, since $X$ compact, so $M(X, P)$ must be compact as well. We have

$$
M(X) \triangleleft_{M(X, P)} P .
$$

By compactness of $M(X, P)$ there is a f.e. $F \subseteq P$ with $M(X) \triangleleft_{M(X, P)} F$. Hence $P \triangleleft_{M(X)} F$, but then also $P_{*} \subseteq F_{*}$. Thus $P$ has a f.e. point-wise subcover $F$. This shows that $X$ is covering compact.

\section{Acknowledgment}

Part of this work was carried out while visiting Japan Advanced Institute of Science and Technology during February 2008. I am very grateful to Hajime Ishihara for his invitation and his hospitality.

\section{References}

[1] E. Bishop and D.S. Bridges, Constructive Analysis, Springer 1985.

[2] P.T. Johnstone, Stone Spaces, Cambridge University Press 1982.

[3] W. Julian, and F. Richman, A uniformly continuous function on [0, 1] that is everywhere different from its infimum, Pacific J. Mathematics 111 (1984), 333 - 340.

[4] E. Palmgren. A constructive and functorial embedding of locally compact metric spaces in locales, Topology and its Applications, 154 (2007), 1854 - 1880; doi:10.1016/j.topol.2007.01.018.

[5] E. Palmgren, Resolution of the uniform lower bound problem in constructive analysis, Mathematical Logic Quarterly 54 (2008), 65 - 69; doi:10.1002/malq.200710034.

[6] G. Sambin, Intuitionistic formal spaces - a first communication, in: D. Skordev (ed.), Mathematical logic and its Applications, Plenum Press 1987, pp. 187 - 204. 
[7] E. Specker, Der Satz vom Maximum in der Rekursive Analysis, Constructivity in Mathematics Proc. Colloq. Amsterdam 1957, 254-265, North-Holland, 1959.

[8] S. Vickers, Localic completion of generalized metric spaces I, Theory and Applications of Categories 14(2005), 328 - 356.

[9] S. Vickers. Localic completion of generalized metric spaces II, Journal of Logic and Analysis 1:11(2009), 1 - 49; doi:10.4115/jla.2009.1.11.

Department of Mathematics, Uppsala University, PO Box 480, S-75106 Uppsala, Sweden

palmgren@math.uu.se

www.math.uu.se

Received: 2 February 2009 Revised: 7 January 2010 